\title{
Shining a light on perioperative Takotsubo syndrome
}

\author{
Eugene A. Hessel II, MD, FACS
}

Received: 2 August 2021 / Revised: 2 August 2021/Accepted: 3 August 2021 / Published online: 27 September 2021

(C) Canadian Anesthesiologists' Society 2021

Recently, Ong et al. wrote a compelling editorial outlining the "Emergence from the shadows" of Takotsubo syndrome (TS), or stress cardiomyopathy, highlighting recent advances in our understanding of the pathophysiology of the disease in recent decades. ${ }^{1}$ Takotsubo syndrome was first described over 30 years ago, and the occurrence of this syndrome in the perioperative period was recognized about ten years later. In subsequent reports, 3-17\% of TS cases were associated with surgical procedures, as reviewed in the Journal in 2016. ${ }^{2}$ Nevertheless, our understanding of TS in the perioperative period remains "in the shadows". More than 15 case reports and series have been published in the past five years, and recent literature reviews have included 28 cases associated with head and neck and maxillofacial surgery, ${ }^{3}$ and four cases associated with liver transplantation. ${ }^{4}$ Despite these recent additions to the literature, we require more information about perioperative $\mathrm{TS}^{5}$ In this issue of the Journal, Garcia Guzzo, et al. ${ }^{6}$ provide an important contribution to the literature, in what is likely the largest report of a single centre experience with perioperative TS.

In their report on 21 cases encountered between 2008 and 2017, Garcia Guzzo et al. noted a number of features in their patients with perioperative TS, with similar results to previous reviews of perioperative TS cases. ${ }^{2,7}$ They go on

This article was updated to correct the "Years covered" in the first column of the table to 2008-2017.

E. A. Hessel II, MD, FACS ( $\square)$

Departments of Anesthesiology and Cardiothoracic Surgery, University of Kentucky College of Medicine, Lexington, KY, USA

e-mail: ehessel@uky.edu to identify key differences between perioperative and nonperioperative cases of TS. Perioperative TS occurred in about one in 15,000 of their surgical cases, which was about half the incidence suspected in an earlier review, ${ }^{2}$ and these cases accounted for $42 \%$ of all physically induced TS in their hospital. In their population, the mean age was $74 \mathrm{yr}, 24 \%$ were men and $91 \%$ had received general anesthesia. Takotsubo syndrome occurred across a range of surgical procedures, and notably $60 \%$ were elective and $49 \%$ considered low- or medium-risk surgery. Only 29\% occurred in high-risk (American Society of Anesthesiologists Physical Status IV) patients. Takotsubo syndrome presented during surgery in $29 \%$, within the first three days in $33 \%$, and beyond three days in $38 \%$ of patients. Presentations included congestive heart failure $(57 \%)$, ST elevation $(43 \%)$, and syncope or cardiac arrest $(10 \%)$. While the initial average left ventricular ejection fraction (LVEF) at time of diagnosis was 35\%, the vast majority (90\%) had complete recovery of LV function. Severe complications occurred in $86 \%$, median stay in the intensive care unit was eight days, and $10 \%$ died, although none from cardiogenic causes. Interestingly, compared with patients with non-perioperative TS at their institution, those with perioperative TS were more commonly men, had lower ejection fractions, received increased inotropes, had higher need for mechanical ventilation, and a longer hospital stay, although mortality rates were similar.

The authors clearly identified the limitations of their study, which include the retrospective study design and some incomplete data. In addition, as their hospital is a tertiary referral centre, they were unable to provide information about the long-term outcome of their patients, nor of the outcome of any subsequent surgical procedures. 
Table Comparison of studies on perioperative Takotsubo syndrome

\begin{tabular}{|c|c|c|c|c|}
\hline & Garcia Guzzo $^{6}$ & Hessel $^{2}$ & Agarwal $^{7}$ & Templin $^{8}$ \\
\hline Year published & 2021 & 2016 & 2017 & 2015 \\
\hline Study design & $\begin{array}{l}\text { Retrospective, Single } \\
\text { centre }\end{array}$ & $\begin{array}{l}\text { Retrospective, case } \\
\text { review }\end{array}$ & $\begin{array}{l}\text { Retrospective, case } \\
\text { review }\end{array}$ & $\begin{array}{l}\text { Prospective, } \mathrm{TS} \\
\text { registry }\end{array}$ \\
\hline Population & Periop TS & Periop TS & Periop TS & All TS \\
\hline Cases $(n)$ & 21 & 131 & 102 & 1,750 \\
\hline Years covered & 2008-2017 & 2000--2016 & 2004-2015 & 1998-2014 \\
\hline Mean or median age $(\mathrm{yr})$ & 74 & 54 & 56 & 67 \\
\hline Females $(\%)$ & 76 & 80 & 84 & 90 \\
\hline $\begin{array}{l}\text { Mean or median LVEF }(\%) \text { on } \\
\text { presentation }\end{array}$ & 35 & 31 & 30 & 41 \\
\hline \multicolumn{5}{|l|}{ Pattern of RWMA } \\
\hline Apical ballooning (\%) & 52 & 68 & 82 & 82 \\
\hline Inverted $(\%)$ & 10 & 8 & 9 & 2 \\
\hline In-hospital mortality (\%) & 10 & 6 & 2 & 4 \\
\hline
\end{tabular}

$\mathrm{LVEF}=$ left ventricular ejection fraction; periop = perioperative; RWMA = regional wall motion abnormality; TS = Takotsubo syndrome.

Several important differences can be seen when comparing perioperative TS and non-perioperative TS. The Table compares Garcia Guzzo et al.'s results with two large case series of perioperative TS and an international registry of all types of TS. Several consistent differences between perioperative TS patients and other cases are seen and these include a higher number of male patients, a higher prevalence of the inverted (basal) pattern of regional wall motion abnormality, a lower initial ejection fraction, and a higher mortality rate. Gibson et al. previously discussed the non-apical variety of perioperative TS. ${ }^{9}$

The high prevalence of malignancy in perioperative TS (38\%) reported by Garcia Guzzo et al. ${ }^{6}$ is novel, and although the association of malignancy with TS is now well-recognized, this association has not previously been reported with perioperative TS specifically. In the International Takotsubo Registry, $16.6 \%$ of patients had diagnosis of malignancy, which was more frequently precipitated by physical stressors. ${ }^{10}$ Another recent publication from Australia reported a similar incidence of antecedent cancer (16.8\%), and further found an association with higher initial clinical severity, and higher long-term mortality, both cardiovascular-related and all-cause mortality. ${ }^{11}$

Ong et al. recently reviewed the remarkable advances in our knowledge about TS from the past decade. ${ }^{1}$ The authors conclude that the syndrome represents a spectrum of neurogenic stunned myocardium. As the principle pathologic feature is myocardial inflammation and edema, often leading to fibrosis, Ong et al. suggest that cardiac magnetic resonance imaging (MRI) be the new diagnostic "gold standard". Notably, unlike earlier suggestions that TS is a benign and transient condition, we now recognize that TS patients often have a complicated early course and prolonged recovery, with some patients never returning to baseline status. Ong et al. also hypothesize that perioperative TS is likely under-recognized and highlight the need for further prospective studies.

Biomarkers may offer a clue to understanding the pathophysiology of the disease, and potential therapeutic targets. Recently, elevated levels of circulating microRNAs miR-16 and miR-26a have been found in patients with TS, as well as in patients with stress, anxiety, and depression, but not in patients with acute myocardial infarction. In animal models, these micro RNAs sensitize the myocardium to TS-like changes when exposed to adrenaline. $^{12}$ MicroRNAs may therefore offer a clue towards identifying at-risk patients, and the basis for developing preventive and therapeutic targets.

The diagnosis and management of TS remains challenging. ${ }^{1,2,13}$ Clinicians may be hesitant to proceed with coronary angiography, and emergency cardiac MRI is logistically difficult or impossible in most centres. A high index of suspicion is crucial, as surgical patients rarely present with classical angina, but more commonly with evidence of heart failure (pulmonary edema or hypotension), electrocardiogram (ECG) changes, arrhythmias, and even cardiac arrest. Early echocardiography imaging is essential, and Ong et al. proposed an algorithm for the presumptive diagnosis of TS, utilizing a combination of a priori probability, ECG changes, hemodynamic status, troponin, and N-terminal pro B-type natriuretic peptide (NT-proBNP). ${ }^{1}$ Others have proposed different diagnostic algorithms, ${ }^{14}$ and using a high ratio of NT-proBNP to troponin to differentiate TS from acute myocardial infarction. ${ }^{15}$ Management of 
cardiac failure in TS is also controversial. ${ }^{14}$ Because of the presumed role of catecholamine excess in the pathogenesis of TS, most experts recommend avoiding exogenous catecholamines to avoid exacerbating myocardial injury. ${ }^{1}$ Ong et al. favour emphasis of volume expansion and speculate that levosimendan may be the inotrope of choice, ${ }^{1}$ while others recommend vasopressin. Nevertheless, vasopressors were used successfully in the study by Garcia Guzzo et al. ${ }^{6}$ and another case review. ${ }^{2}$ Although inotropes may be associated with worse outcome, this may instead reflect severe cardiac damage. Finally, many experts recommend early initiation of mechanical circulatory assistance if higher doses of inotropes are required.

The study by Garcia Guzzo et al. is an important contribution to our knowledge about perioperative TS. These results highlight remaining gaps and opportunities for further investigation, to which we hope our readers will respond. The precise incidence of TS in patients undergoing anesthesia for surgical procedures is unknown, and the true incidence may have been underestimated. Further analyses from other institutions would allow us to estimate the incidence, presentation, and outcome more precisely. Another unresolved question is why some patients experience TS and others do not, despite similar levels of stress. Case control studies of surgical patients with and without TS might provide clues. A third unresolved question is optimal management of cardiac failure including choice of vasopressors and inotropes. Finally, we lack data on the optimal perioperative management of patients with a past medical history of TS. Future research on this condition would benefit from the establishment of a national or international perioperative TS registry to increase access to patients with this uncommon condition and improve generalizability.

\section{Un nouvel éclairage sur le syndrome périopératoire de Takotsubo}

Récemment, Ong et coll. ont rédigé un éditorial convaincant décrivant «L'émergence de l'ombre »du syndrome de Takotsubo, ou cardiomyopathie de stress, soulignant les progrès récents dans notre compréhension de la physiopathologie de la maladie au cours des dernières décennies. ${ }^{1}$ Le syndrome de Takotsubo a été décrit pour la première fois il y a plus de 30 ans, et l'apparition de ce syndrome en période périopératoire a été reconnue environ dix ans plus tard. Dans les comptes rendus ultérieurs, 3 à 17 $\%$ des cas de syndromes de Takotsubo étaient associés à des interventions chirurgicales, comme cela a été rapporté dans le Journal en 2016. ${ }^{2}$ Néanmoins, notre compréhension du syndrome de Takotsubo en période périopératoire demeure «dans l'ombre ». Plus de 15 cas cliniques et séries de cas ont été publiés au cours des cinq dernières années, et les revues de littérature récentes ont inclus 28 cas associés à des chirurgies de la tête, du cou et maxillo-faciales, ${ }^{3}$ ainsi que quatre cas associés à une transplantation hépatique. ${ }^{4}$ Malgré ces ajouts récents à la littérature, nous avons besoin de plus d'informations sur le syndrome de Takotsubo périopératoire. ${ }^{5}$ Dans ce numéro du Journal, Garcia Guzzo et coll. ${ }^{6}$ font une contribution majeure à la littérature, dans ce qui est probablement le plus important compte rendu de l'expérience d'un seul centre avec le syndrome de Takotsubo périopératoire.

Dans leur compte rendu de 21 cas observés entre 2008 et 2017, Garcia Guzzo et coll. ont noté un certain nombre de caractéristiques chez leurs patients atteints d'un syndrome de Takotsubo périopératoire, avec des résultats similaires aux comptes rendus précédents de cas de syndromes de Takotsubo périopératoires. ${ }^{2,7}$ Les auteurs identifient ensuite les principales différences entre les cas périopératoires et non périopératoires de syndrome de Takotsubo. Un syndrome de Takotsubo périopératoire est survenu dans environ un cas chirurgical sur 15000 , soit environ la moitié de l'incidence suspectée dans un rapport antérieur, ${ }^{2}$ et ces cas représentaient $42 \%$ de tous les syndromes de Takotsubo d'origine physique dans leur hôpital. Dans la population étudiée, l'âge moyen était de 74 ans, $24 \%$ étaient des hommes et $91 \%$ avaient reçu une anesthésie générale. Le syndrome de Takotsubo est survenu dans plusieurs types d'interventions chirurgicales; fait remarquable, $60 \%$ sont survenus dans des cas non urgents et $49 \%$ dans des cas considérés comme chirurgie à risque faible ou moyen. Seulement $29 \%$ des cas sont survenus chez des patients à haut risque (score IV de l'American Society of Anesthesiologists). Le syndrome de Takotsubo s'est présenté pendant la chirurgie chez $29 \%$ des patients, au cours des trois premiers jours chez $33 \%$, et après plus de trois jours chez $38 \%$ des patients. Les présentations comprenaient l'insuffisance cardiaque congestive (57\%), l'élévation du segment ST (43\%) et la syncope ou l'arrêt cardiaque (10\%). Alors que la fraction d'éjection ventriculaire gauche (FEVG) moyenne initiale au moment du diagnostic était de $35 \%$, la grande majorité des patients $(90 \%)$ ont complètement récupéré leur fonction VG. Des complications graves sont survenues chez $86 \%$ des patients, le séjour médian à l'unité de soins intensifs était de huit jours, et $10 \%$ sont décédés, bien qu'aucun patient ne soit décédé de causes cardiogéniques. Fait intéressant, par rapport aux patients atteints d'un syndrome de Takotsubo non périopératoire dans leur établissement, les patients atteints d'un syndrome de Takotsubo périopératoire étaient plus souvent des 
Tableau Comparaison des études sur le syndrome de Takotsubo périopératoire

\begin{tabular}{|c|c|c|c|c|}
\hline & Garcia Guzzo $^{6}$ & Hessel $^{2}$ & Agarwal $^{7}$ & Templin $^{8}$ \\
\hline Année de publication & 2021 & 2016 & 2017 & 2015 \\
\hline Conception de l'étude & $\begin{array}{l}\text { Rétrospective, } \\
\text { monocentrique }\end{array}$ & $\begin{array}{l}\text { Rétrospective, compte } \\
\text { rendu de cas }\end{array}$ & $\begin{array}{l}\text { Rétrospective, compte } \\
\text { rendu de cas }\end{array}$ & $\begin{array}{l}\text { Prospective, registre du } \\
\text { syndrome de Takotsubo }\end{array}$ \\
\hline Population & $\begin{array}{l}\text { syndrome de Takotsubo } \\
\text { périopératoire }\end{array}$ & $\begin{array}{l}\text { syndrome de Takotsubo } \\
\text { périopératoire }\end{array}$ & $\begin{array}{l}\text { syndrome de Takotsubo } \\
\text { périopératoire }\end{array}$ & $\begin{array}{l}\text { tous les syndromes de } \\
\text { Takotsubo }\end{array}$ \\
\hline Cas $(n)$ & 21 & 131 & 102 & 1750 \\
\hline Années couvertes & 2008-2017 & 2000--2016 & 2004-2015 & 1998-2014 \\
\hline Âge moyen ou médian (année) & 74 & 54 & 56 & 67 \\
\hline Femmes $(\%)$ & 76 & 80 & 84 & 90 \\
\hline $\begin{array}{l}\text { FEVG moyenne ou médiane } \\
(\%) \text { à la présentation }\end{array}$ & 35 & 31 & 30 & 41 \\
\hline \multicolumn{5}{|l|}{ Cinétique de l'ARC de la paroi } \\
\hline Ballonnement apical (\%) & 52 & 68 & 82 & 82 \\
\hline Inversé (\%) & 10 & 8 & 9 & 2 \\
\hline Mortalité hospitalière (\%) & 10 & 6 & 2 & 4 \\
\hline
\end{tabular}

$\mathrm{FEVG}$ = fraction d'éjection ventriculaire gauche; $\mathrm{ARC}=$ anomalie régionale de contractilité

hommes, avaient des fractions d'éjection plus faibles, ont reçu plus d'inotropes, présentaient des besoins plus élevés de ventilation mécanique et ont séjourné plus longtemps à l'hôpital, bien que les taux de mortalité entre les deux groupes soient similaires.

Les auteurs ont clairement identifié les limites de leur étude, qui comprennent sa nature rétrospective et certaines données incomplètes. De plus, comme leur hôpital est un centre de référence tertiaire, les auteurs n'ont pas été en mesure de fournir des informations sur les issues à long terme de leurs patients, ni sur les issues de toute intervention chirurgicale ultérieure.

Plusieurs différences importantes peuvent être observées lors de la comparaison du syndrome de Takotsubo périopératoire et non périopératoire. Le tableau compare les résultats de Garcia Guzzo et coll. à deux importantes séries de cas de syndromes de Takotsubo périopératoires et un registre international de tous les types de syndromes de Takotsubo. Plusieurs différences récurrentes entre les patients atteints de syndrome de Takotsubo périopératoire et les autres cas sont décrites, notamment un nombre plus élevé de patients de sexe masculin, une prévalence plus élevée de la cinétique inversée (basale) d'anomalie régionale de contractilité de la paroi, une fraction d'éjection initiale plus faible, et un taux de mortalité plus élevé. Gibson et coll. ont déjà discuté de la variété non apicale de syndrome de Takotsubo périopératoire. ${ }^{9}$

La forte prévalence de malignité dans le syndrome de Takotsubo périopératoire $(38 \%)$ rapportée par Garcia Guzzo et coll. ${ }^{6}$ est nouvelle et, bien que l'association entre malignité et syndrome de Takotsubo soit maintenant bien reconnue, cette association n'a pas encore été rapportée spécifiquement en cas de syndrome de Takotsubo périopératoire. Dans le Registre international de Takotsubo, 16,6\% des patients ont reçu un diagnostic de malignité, lequel était plus fréquemment précipité par des facteurs de stress physiques. ${ }^{10}$ Une autre publication australienne récente a rapporté une incidence similaire d'antécédent de cancer $(16,8 \%)$, et a en outre noté une association avec une gravité clinique initiale plus élevée et une mortalité à long terme, à la fois cardiovasculaire et toutes causes confondues, plus élevée. ${ }^{11}$

Ong et coll. ont récemment passé en revue les progrès remarquables de nos connaissances concernant le syndrome de Takotsubo au cours de la dernière décennie. ${ }^{1}$ Les auteurs concluent que le syndrome représente un spectre de sidération myocardique neurogénique. L'inflammation du myocarde et l'œdème constituant la principale caractéristique pathologique, ce qui mène souvent à la fibrose, Ong et coll. suggèrent que l'imagerie par résonance magnétique (IRM) cardiaque devienne le nouvel «étalon-or» du diagnostic. Fait notable, contrairement aux suggestions précédentes selon lesquelles le syndrome de Takotsubo serait une affection bénigne et transitoire, nous reconnaissons aujourd'hui que, chez les patients atteints de ce syndrome, l'évolution précoce est souvent compliquée et le rétablissement prolongé, certains patients ne récupérant jamais leur état de base. Ong et coll. émettent également l'hypothèse que le syndrome de Takotsubo périopératoire est probablement sous-diagnostiqué et soulignent la nécessité d'études prospectives supplémentaires.

Les biomarqueurs pourraient être un indice pour comprendre la physiopathologie de la maladie et ses 
cibles thérapeutiques potentielles. Récemment, des niveaux élevés de micro-ARN miR-16 et miR-26a circulants ont été observés chez des patients atteints de syndrome de Takotsubo, ainsi que chez des patients souffrant de stress, d'anxiété et de dépression, mais pas chez des patients atteints d'infarctus aigu du myocarde. Dans les modèles animaux, ces micro-ARN sensibilisent le myocarde aux changements similaires au syndrome de Takotsubo lorsque le myocarde est exposé à l'adrénaline. ${ }^{12}$ Les micro-ARN pourraient donc constituer une piste de compréhension pour identifier les patients à risque, et servir de base pour mettre au point des cibles de prévention et de traitement.

Le diagnostic et la prise en charge du syndrome de Takotsubo demeurent difficiles. ${ }^{1,2,13}$ Les cliniciens peuvent être réticents à l'idée de procéder à une coronarographie et, dans la plupart des centres, l'IRM cardiaque d'urgence est difficile, voire impossible à réaliser d'un point de vue logistique. Un indice de suspicion élevé est crucial, car les patients chirurgicaux présentent rarement une angine classique, mais plus souvent des signes d'insuffisance cardiaque (œdème pulmonaire ou hypotension), des changements d'électrocardiogramme (ECG), des arythmies et même des signes d'arrêt cardiaque. L'imagerie par échocardiographie précoce est essentielle, et Ong et coll. ont proposé un algorithme pour poser un diagnostic présomptif de syndrome de Takotsubo. Cet algorithme se fonde sur une combinaison de probabilité a priori, de changements au niveau de l'ECG, de statut hémodynamique, de troponine et de prohormone $\mathrm{N}$-terminale du peptide natriurétique cérébral (NTproBNP). ${ }^{1}$ D'autres ont proposé différents algorithmes de diagnostic, ${ }^{14}$ et l'utilisation d'un rapport élevé de NTproBNP à la troponine afin de différencier le syndrome de Takotsubo de l'infarctus aigu du myocarde. ${ }^{15}$ La prise en charge de l'insuffisance cardiaque lors d'un syndrome de Takotsubo est également controversée. ${ }^{14}$ En raison du rôle présumé de l'excès de catécholamine dans la pathogenèse du syndrome de Takotsubo, la plupart des experts recommandent d'éviter les catécholamines exogènes pour ne pas exacerber la lésion myocardique. ${ }^{1}$ Ong et coll. préfèrent mettre l'accent sur l'expansion du volume et spéculent que le lévosimendan pourrait être l'inotrope de choix, ${ }^{1}$ tandis que d'autres recommandent plutôt la vasopressine. Néanmoins, les vasopresseurs ont été utilisés avec succès dans l'étude de Garcia Guzzo et coll. ${ }^{6}$ et un autre cas clinique. ${ }^{2}$ Bien que les inotropes puissent être associés à des issues moins favorables, cela pourrait plutôt être le reflet de graves lésions cardiaques. Enfin, de nombreux experts recommandent l'amorce précoce d'une assistance circulatoire mécanique si des doses plus élevées d'inotropes sont nécessaires.

L'étude de Garcia Guzzo et coll. constitue une importante contribution à nos connaissances sur le syndrome de Takotsubo périopératoire. Ces résultats mettent en évidence les lacunes qui subsistent et les possibilités de recherches plus approfondies, auxquelles nous espérons que nos lecteurs répondront. L'incidence précise du syndrome de Takotsubo chez les patients recevant une anesthésie pour des interventions chirurgicales est inconnue, et l'incidence réelle pourrait avoir été sous-estimée. D'autres analyses provenant d'autres institutions nous permettraient d'estimer plus précisément son incidence, sa présentation et ses issues. Une autre question non résolue est de savoir pourquoi certains patients développent un syndrome de Takotsubo et d'autres non, malgré des niveaux de stress similaires. Des études cas témoins de patients chirurgicaux avec et sans syndrome de Takotsubo pourraient fournir des indices. Une troisième question non résolue touche à la prise en charge optimale de l'insuffisance cardiaque, y compris au choix des vasopresseurs et des inotropes. Enfin, nous manquons de données sur la prise en charge périopératoire optimale des patients ayant des antécédents médicaux de syndrome de Takotsubo. Les recherches futures sur cette maladie bénéficieraient de la création d'un registre national ou international de syndromes de Takotsubo périopératoires afin d'accroître l'accès aux patients atteints de cette affection rare et d'améliorer la généralisabilité.

Disclosures None.

Funding statement None.

Editorial Responsibility This submission was handled by Dr. Alana M. Flexman, Associate Editor, Canadian Journal of Anesthesia.

Déclaration Aucune.

Déclaration de financement Aucune.

Responsabilité éditoriale Cet article a été traité par Dre Alana M Flexman, rédactrice adjointe, Journal canadien d'anesthésie.

\section{References}

1. Ong GJ, Nguyen TH, Kucia A, et al. Takotsubo syndrome: finally emerging from the shadows? Heart Lung Circ 2021; 30: 36-44.

2. Hessel EA 2nd. Takotsubo cardiomyopathy and its relevance to anesthesiology: a narrative review. Can J Anesth 2016; 63: 1059-74.

3. Brooks JK, Warburton G, Clark BC. Takotsubo syndrome after surgical and nonsurgical oral and maxillofacial events: review of published cases. J Oral Maxillofac Surg 2019; 77: 478-88.

4. Vitin AA, Azamfirei L, Tomescu D. Perioperative stress-induced (Takotsubo) cardiomyopathy in liver transplant recipients. J Crit Care Med (Targu Mures) 2018; 4: 56-63. 
5. Hessel EA 2nd, London MJ. Takotsubo (stress) cardiomyopathy and the anesthesiologist: enough case reports. Let's try to answer some specific questions! Anesth Analg 2010; 110: 674-9.

6. Garcia Guzzo ME, Novas DS, Iglesias FA, Deluca D, Domenech $G$, Terrasa SA. Anesthetic implications of perioperative Takotsubo syndrome: a retrospective cohort study. Can J Anesth 2022; this issue. DOI: https://doi.org/10.1007/s12630021-02109-9.

7. Agarwal S, Bean MG, Hata JS, Castresana MR. Perioperative Takotsubo cardiomyopathy: a systematic review of published cases. Semin Cardiothorac Vasc Anesth 2017; 21: 277-90.

8. Templin C, Ghadri JR, Diekmann J, et al. Clinical features and outcomes of takotsubo (stress) cardiomyopathy. N Engl J Med 2015; 373: 929-38.

9. Gibson LE, Klinker MR, Wood JM. Variants of Takotsubo syndrome in the perioperative period: a review of potential mechanisms and anaesthetic implications. Anaesth Crit Care Med 2020; 39: 647-54.

10. Cammann VL, Sarcon A, Ding KJ, et al. Clinical features and outcomes of patients with malignancy and takotsubo syndrome: observations from the International Takotsubo Registry. J Am Heart Assoc 2019; DOI: https://doi.org/10.1161/JAHA.118. 010881.
11. Nguyen TH, Stansborough J, Ong GJ, Surikow S, Price TJ, Horowitz JD. Antecedent cancer in Takotsubo syndrome predicts both cardiovascular and long-term mortality. Cardiooncology 2019; DOI: https://doi.org/10.1186/s40959-019-0053-6.

12. Couch LS, Fiedler J, Chick G, et al. Circulating microRNAs predispose to takotsubo syndrome following high-dose adrenaline exposure. Cardiovasc Res 2021; DOI: https://doi.org/10.1093/ $\mathrm{cvr} / \mathrm{cvab} 210$

13. Agarwal S, Sanghvi C, Odo N, Castresana MR. Perioperative takotsubo cardiomyopathy: implications for anesthesiologist. Ann Card Anaesth 2019; 22: 309-15.

14. Ghadri JR, Wittstein IS, Prasad A, et al. International expert consensus document on takotsubo syndrome (part II): diagnostic workup, outcome, and management. Eur Heart J 2018; 39: 2047-62.

15. Gopalakrishnan $P$, Zaidi $R$, Sardar MR. Takotsubo cardiomyopathy: pathophysiology and role of cardiac biomarkers in differential diagnosis. World J Cardiol 2017; 9: 723-30.

Publisher's Note Springer Nature remains neutral with regard to jurisdictional claims in published maps and institutional affiliations. 